\title{
Les entreprises aérospatiales de défense : quelles cohérences technologiques de leur R\&D?
}

\section{Aerospace defense companies: what are the technological coherences of their R\&D?}

\author{
Cécile Fauconnet ${ }^{1}$, Didier Lebert ${ }^{2}$, Célia Zyla ${ }^{3}$, Sylvain Moura ${ }^{4 *}$ \\ ${ }^{1}$ Unité d'Economie Appliquée, ENSTA ParisTech, France, cecile.fauconnet@ensta-paristech.fr \\ ${ }^{2}$ Unité d'Economie Appliquée, ENSTA ParisTech, France, didier.lebert@ensta-paristech.fr \\ ${ }^{3}$ SATT Lutech, celia.zyla@sattlutech.com \\ ${ }^{4}$ Observatoire Economique de la Défense, Ministère des Armées, sylvain.moura@intradef.gouv.fr
}

RÉSUMÉ. En dépit des enjeux économiques et de sécurité que porte la base industrielle et technologique de défense (BITD) française et en particulier celles de l'aéronautique de défense (AD), celle-ci a été peu caractérisée du point de vue de ses technologies (production et usage). Notre article vise à combler en partie ce vide. Nous nous intéressons à l'organisation de la R\&D des entreprises $A D$ et à leur production d'innovations via une approche "brevets ". Nous proposons une typologie de ces entreprises selon leur capacité à engendrer des synergies technologiques dans leur activité d'innovation et selon les arbitrages qu'elles font entre innovations d'exploration et innovations d'exploitation.

ABSTRACT. Despite the economic and security challenges related to the French defense technological and industrial base, in particular for defense aeronautics (DA), this base has been poorly characterized from the point of view of its technologies (production and use). Our article aims to fill some of this gap. We will focus on the organization of the R\&D of DA companies and their production of innovations via a "patent" approach. We propose a typology of those companies according to their capacity to generate technological synergies in their innovation activity and trade-offs they make between exploration innovations and exploitation innovations.

MOTS-CLÉS. Base industrielle et technologique de défense, aérospatial, recherche et développement, brevets, cohérence technologique, dilemme exploration exploitation.

KEYWORDS. French defence technological and industrial base, aerospace, research and development, patents, technological coherence, exploration-exploitation dilemma.

\section{Introduction}

Les entreprises aéronautiques de défense ont une activité intensive en recherche et développement $(\mathrm{R} \& \mathrm{D})$. Elles cherchent à repousser les frontières technologiques. En cela, elles répondent à la volonté de la politique de défense de satisfaire l'autonomie stratégique pour les armées nationales [MIN 13]. Les programmes d'armement, éléments structurants de l'industrie de défense, reposent grandement sur des considérations de maîtrise de technologies [SER 14]. La R\&D répond aussi à la politique publique d'innovation qui confère aux entreprises de défense un rôle d'entraîneur technologique [SER 08]. Dans le même temps, elle leur permet de gagner en compétitivité sur les marchés, notamment d'exportation [FON 14].

En dépit des enjeux économiques et de sécurité que porte la base industrielle et technologique de défense (BITD) française et en particulier celles de l'aéronautique de défense (AD), celle-ci a été peu caractérisée du point de vue de ses technologies (production et usage). L'intérêt a surtout porté sur les conditions du financement des entreprises de défense [BEL 08] [OUD 15]. Seule une étude cas sur le groupe Thales [AYE 08] déploie une approche par la technologie, encore que le propos soit surtout centré sur la gestion des droits de propriété en découlant.

\footnotetext{
* Les opinions présentées ici sont celles de l'auteur et ne sauraient engager le Ministère des Armées. 
Notre article vise à analyser les entreprises aéronautiques de défense sous l'angle de leurs technologies. Nous allons nous intéresser à l'organisation de leur R\&D et à la production d'innovations via une approche «brevets ». Deux thèmes sont plus précisément abordés pour d'identifier certaines spécificités de ces entreprises. Premièrement, quelles relations entretiennent-elles entre leur dualité technologique (leur capacité à produire des briques technologiques militaires et civiles) et leur dualité productive (leur capacité à engendrer du chiffre d'affaires militaire et civil) ? Deuxièmement, quels liens établissent-elles entre leur diversité technologique (le nombre de briques de connaissances technologiques qu'elles produisent) et la nature de leur R\&D (orientée plutôt vers l'exploration d'architectures de connaissances technologiques originales, ou vers l'exploitation d'architectures maîtrisées) ?

Ce faisant, il s'agit de répondre à la question suivante : alors même que ces entreprises sont diverses - en termes de taille, d'activités, de structure et stratégie de R\&D, etc. -, quelles organisations technologiques développent-elles dans leur activité d'innovation ? Nous introduisons une mesure multidimensionnelle de leur cohérence technologique [NES 05] et en déduisons une typologie des entreprises de la BITD dans le domaine AD selon leur capacité à engendrer des synergies dans leur activité d'innovation, selon les arbitrages qu'elles font entre innovations d'exploration et d'exploitation, les technologies qu'elles manipulent (en nombre et en étendue), leur taille, leur taux de dépendance à la défense, etc. Les entreprises aéronautiques de défense sont repérées grâce à leur code $\mathrm{APE}^{1}$ et à leur participation à des programmes aéronautiques militaires.

La section 2 de l'article pose les hypothèses de travail en lien avec les deux thèmes décrits plus haut: la relation entre dualité technologique et dualité productive d'une part, celle entre diversité technologique et gestion du dilemme exploration / exploitation d'autre part. La section 3 fait un point sur les mesures de la cohérence technologique et du ratio d'exploitation. La section 4 présente l'échantillon d'entreprises issu de la BITD et les données qui lui sont associées. La dernière section est consacrée aux principaux résultats de l'étude.

\section{Les hypothèses sur la relatedness et la cohérence technologique des entreprises AD}

La relation entre dualité d'activités et dualité technologique peut être abordée dans un cadre d'analyse qui relève, en économie industrielle et en management stratégique, du lien entre la diversification ou le recentrage avec la performance économique et financière des entreprises (même si nous n'envisageons pas dans notre article d'associer cette relation à la performance).

Dans la droite ligne des travaux d'Ansoff [ANS 65], la proposition selon laquelle la diversification conglomérale assure à l'entreprise une minimisation de ses risques systématiques est questionnée au regard de l'impact potentiellement négatif pouvant exister entre cette stratégie et la performance de l'entreprise. Ansoff met en avant la nécessité d'associer la pratique de la diversification à l'état donné du portefeuille d'activités afin de maximiser les synergies productives et commerciales. Il existe donc deux stratégies de diversification ayant un effet théorique différencié sur la performance de la firme : la diversification conglomérale, ou «non liée », versus la diversification «liée ». Comme le montre [ELY 13], la stratégie de diversification (resp. la stratégie de recentrage) liée peut ne pas être marquée par une hausse (resp. une baisse) du nombre d'activités dans le portefeuille de l'entreprise. Ces stratégies possèdent ainsi deux dimensions qui peuvent être dissociées : l'évolution du nombre d'activités - et l'on parle alors de diversification ou de recentrage « quantitatif » - et l'évolution de l'intensité des liaisons entre activités - et l'on parle alors de diversification ou de recentrage « qualitatif ».

La question de la mesure de l'intensité de la liaison entre les activités productives de l'entreprise a donné lieu à une abondante littérature. La manière la plus courante de procéder consiste à calculer des distances entre les activités d'un même portefeuille selon leur appartenance à des champs plus ou moins identiques d'une nomenclature d'activités externe. Par exemple, [PAL 85] utilise la 
nomenclature d'activités américaine SIC (Standard Industrial Classification) en considérant comme fortes les liaisons entre les activités qui appartiennent à des niveaux identiques d'agrégation de cette nomenclature à trois digits, faibles les liaisons entre activités appartenant à des niveaux d'agrégation à deux digits identiques, nulles pour un degré d'agrégation plus amont. Des indices d'entropie spécifiques aux activités liées et non liées d'un portefeuille sont alors calculés (avec le chiffre d'affaires des différents segments servant à pondérer chacune des activités), et la relation théorique testée est celle entre l'intensité des liaisons et la valeur du ROA (rendement des actifs) ou du ROI (retours sur investissement) de l'entreprise.

L'usage des indices d'entropie pour appréhender la liaison est encore répandu aujourd'hui [ELY 14]. D'autres approches ont été proposées par ailleurs. Par exemple, les travaux de Rumelt [RUM 74] [RUM 82] et de Montgomery [MON 79] [MON 94] abordent le sujet de la liaison à travers des catégories stratégiques qui associent le degré de spécialisation de l'entreprise (le poids en termes de chiffre d'affaires de son activité principale) au degré de connexité des activités (selon l'identité des ressources utilisées pour les opérer). Avec cette littérature nous en revenons aux préoccupations premières d'Ansoff pour qui les stratégies de développement des entreprises sont principalement guidées par ce degré de connexité de ressources. C'est ce que montreront empiriquement [CHR 81] pour la diversification, et [KLE 09] pour le recentrage (les entreprises se désengagent prioritairement des activités qui utilisent peu de ressources communes avec celles employées dans leur métier cœur).

La liaison entre activités se conçoit donc ici au niveau des ressources. De multiples entrées ont été explorées, et [TAN 05] tentent d'en faire la synthèse : ressources humaines, technologiques, etc. Concernant la ressource «technologies», trois entrées sont privilégiées. [ROB 95] tout d'abord s'appuient sur les matrices de flux technologiques de Scherer [SCH 82] construites à partir de données de brevets pour calculer l'intensité des liens entre les activités productives. [RON 05] de leur côté, à la suite de [FAN 00], produisent des indices de liaison technologique à partir de données obtenue au niveau macro - issues des Comptes nationaux (Tableaux des Echanges Interindustriels). Les prolongements de l'approche de la cohérence [PIS 00] [PIS 04] (voir section suivante) mobilisent également des données de brevets pour border l'activité d'innovation de l'entreprise. Dans ces trois cas, la liaison «technologies » détermine entièrement la liaison «activités », ce qui fait qu'une relation directe est effectuée entre la première et la performance de l'entreprise.

Chez Robins et Wiersema, Piscitello, ou encore Rondi et Vannoni, la prise en compte de la seule dimension qualitative de la stratégie de développement amène donc à gommer la diversité des activités elles-mêmes. Au mieux, cette diversité est utilisée au numérateur d'un indicateur de relatedness pour normer sa valeur et procéder à des comparaisons entre entreprises à diversité productive différente. La distinction entre dualité technologique et dualité d'activités réclame de réintroduire la dimension quantitative.

Comme on l'anticipe à partir des éléments précédents, cette distinction peut être faite de deux manières différentes : en comptant les activités (diversité au sens strict) ou en raisonnant en parts de chiffres d'affaires (spécialisation au sens de Rumelt). C'est cette deuxième approche que nous retiendrons ici en posant qu'une entreprise de défense ayant une activité «diversifiée » est celle qui possède un ratio de spécialisation dans les activités de défense inférieur à un certain seuil. Elle est alors duale sur le plan de ses activités parce qu'une part significative de son chiffre d'affaires est issue de marchés civils.

Nous retenons la méthode de [ROB 95] pour la dualité technologique: nous privilégions une approche qui s'intéresse à la destination en activités des technologies plutôt qu'à l'organisation des processus R\&D (plus ou moins « cohérente ») telle que Piscitello la conçoit. Plus précisément, de notre point de vue, la cohérence de l'organisation des processus de R\&D des entreprises renvoie à des questions qui ont trait à des choix stratégiques d'une autre nature que ceux de la dualité. Nous y revenons plus bas. 
La dualité technologique rapporte alors le nombre de briques technologiques maîtrisées par l'entreprise qui sont destinées à un usage militaire à l'ensemble des briques technologiques manipulées par la firme. Une entreprise ayant une activité de défense est duale technologiquement si les technologies qu'elle maîtrise comprennent une part significative de technologies hors défense.

Couper le lien exclusif entre la ressource et l'activité productive et commerciale ne signifie pas que toutes les combinaisons «dual / non dual » peuvent se trouver aux mêmes degrés. Nous faisons ainsi l'hypothèse que la dualité technologique est associée positivement à la dualité d'activités. Cette hypothèse résulte d'une lecture des références citées plus haut qui accolent donc strictement les liaisons à ces deux niveaux, jusqu'à les confondre.

[PIS 00] [PIS 04] puis [NES 05] et [KRA 11] partent d'une même littérature initiale, de mêmes questionnements (identifier les liens entre l'effort et la nature de l'innovation de l'entreprise et sa performance) mais en centrant leur réflexion sur la représentation de l'organisation de la R\&D des entreprises. Pour cela ils s'inspirent du modèle de la cohérence de la grande entreprise mis au point par [TEE 94].

[ELY 15] montrent qu'il est possible d'étendre cette représentation pour qualifier l'organisation concrète de la R\&D des firmes en termes de dilemme exploration / exploitation [MAR 91]. Il devient alors possible de revenir aux constats effectués par [QUI 08] pour qui la diversité technologique a un effet plus important sur la capacité exploratoire que sur la capacité d'exploitation de l'entreprise. Qu'en est-il pour les entreprises possédant une activité de défense?

Dans la section suivante nous présentons la manière de calculer la capacité d'exploration d'une entreprise en nous situant en droite ligne de [TEE 94] et des travaux que cet article a inspiré. Comme chez [QUI 08] et chez [BOT 10], la diversité technologique de l'entreprise correspond au nombre de briques technologiques de nature différente qu'elle est en mesure de produire. Avec un biais inhérent à l'approche stricte de la cohérence que l'on retrouve chez les derniers auteurs qui fait que l'on ne peut pas l'appliquer immédiatement : le lien positif entre diversité et cohérence qu'ils mettent en évidence empiriquement repose sur une organisation purement théorique de la $R \& D$ de la firme qui suppose que toutes les liaisons technologiques possibles - entre chaque brique technologique produite - sont mises en œuvre - «exploitées », donc. L'écart entre cette situation d'exploitation maximale et la situation concrète de la R\&D de l'entreprise représente le poids de l'exploration dans cette organisation. L'hypothèse que nous posons - reprenant [QUI 08] - est ainsi que la diversité des briques technologiques produites par l'entreprise s'associe positivement avec cette capacité d'exploration comprise comme un écart entre deux cohérences, l'une théorique, l'autre concrète.

\section{La mesure de la cohérence technologique}

Les mesures de la cohérence introduites par [TEE 94] sont intimement liées à la manière structurale / réticulaire de représenter les bases de connaissance des entreprises [SAV 09]. Cependant, elles ne peuvent parfaitement l'être, car deux entreprises manipulant les mêmes technologies mais les liant de manières différentes auront un même score de cohérence globale et de cohérence de voisinage ( $c f$. infra). C'est la raison pour laquelle nous proposons de compléter l'étude de la cohérence des bases de connaissance des entreprises en articulant :

- la cohérence globale, où toutes les liaisons possibles entre les technologies manipulées par l'entreprise sont prises en compte. Cette cohérence se mesure par l'indicateur WAR - weighted-average relatedness - de [TEE 94] adapté au contexte de la cohérence technologique par [NES 05] ;

- la cohérence de voisinage, ou du « cœur », où seules les associations technologiques les plus fortes sont prises en considération de sorte à former un «arbre couvrant maximal ». Cet indice correspond à WARN - weighted-average relatedness of neighbors - chez [TEE 94] ; 
- la cohérence des relations inter-technologiques concrètes, où seuls les liens réellement identifiés entre technologies au sein de la base de connaissance de l'entreprise sont pris en compte [NAS 13]. Nous nommons cet indicateur KBC (figure 2).

Ce sont par des données de brevets que nous représentons les bases de connaissance des entreprises de manière réticulaire. Notons $T$ le nombre total de technologies différentes dans la base de données de brevets, $p_{k}$ le vecteur de format $T \times \mathbf{1}$ indiquant pour chacun de ses arguments le nombre total d'occurrences des $T$ technologies pour l'entreprise ${ }^{k}, p_{k i}$ ce nombre total pour la technologie $i$, et $t_{k}$ l'étendue technologique de l'entreprise (le nombre de technologies différentes qu'elle produit, i.e. le nombre d'arguments non nuls de $\left.p_{k}\right)$, alors $P_{k} \equiv \sum_{i} p_{k i}$ correspond à l'ensemble des occurrences technologiques dans les brevets détenus par l'entreprise (figure 1).

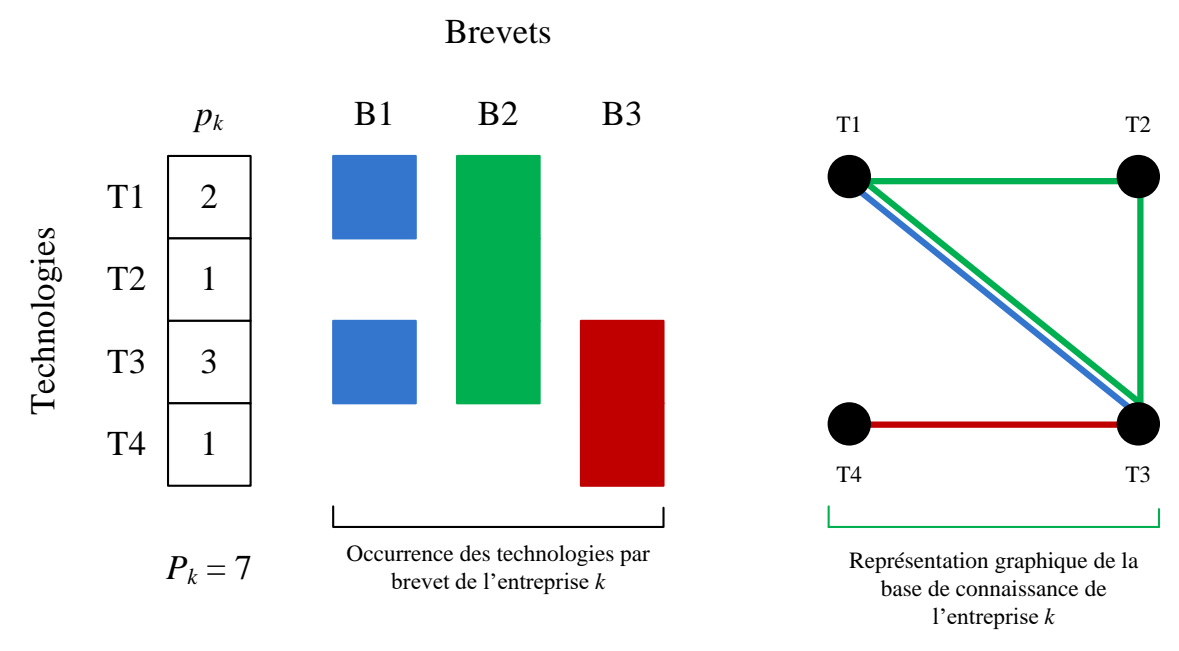

Figure 1. Structure des données pour la mesure de la cohérence technologique d'une entreprise

Appelons $C_{k}$ l'indice de cohérence pour l'entreprise ${ }^{k}$, cet indice pouvant prendre trois formes distinctes selon le contenu de $M^{k}$, matrice booléenne de format $T \times T$ qui résume les liaisons technologiques selon chacune des trois configurations de la cohérence pour l'entreprise ${ }^{k}$. Alors :

$$
C_{k}=\frac{1}{P_{k}} \sum_{i} p_{k i}\left(\frac{\Sigma_{j \neq i} \tau_{i j} M_{i j}^{k} p_{k j}}{\sum_{j \neq i} M_{i j}^{k} p_{k j}}\right)
$$

Chaque entreprise est ainsi agrémentée de trois indicateurs de cohérence en valeur absolue (selon la forme de la matrice $M$ ), et de deux en valeur relative (taux d'exploration et d'exploitation, $c f$. figure 2) :

- pour $W A R$, la matrice adjacente $M$ est uniquement composée de 1 pour intégrer toutes les liaisons possibles entre les technologies manipulées dans la base de connaissance de ${ }^{k}$;

- pour WARN, la matrice $M$ intègre la force des liens technologiques dans l'espace de production des innovations de l'entreprise. Alors que WAR associe les technologies de l'entreprise en $\frac{t_{k}\left(t_{k}-1\right)}{2}$ liens, il s'agit ici de toutes les associer en $t_{k}-\mathbf{1}$ liens seulement (i.e. dans un arbre) en ne retenant que l'association - étant donné les valeurs de $\tau_{i j}$, mesure de cette force [BOT 10] - qui produit la pondération totale de l'arbre la plus forte. Autrement dit, la définition de $M$ repose sur une procédure d'arbre couvrant maximal pondéré (weighted maximum spanning tree) et $M_{i j}^{k}=\mathbf{1}$ si $i j$ appartient à l'arbre couvrant maximal, 0 sinon ; 
- pour $K B C$, la matrice $M$ rend compte des liens concrètement identifiés entre technologies au sein de la base de connaissance de l'entreprise, avec $M_{i j}^{k}=\mathbf{1}$ si la liaison $i j$ appartient effectivement à la base de connaissance de $k, 0$ sinon. C'est l'indicateur de «complémentarité technologique » de [NAS 13].

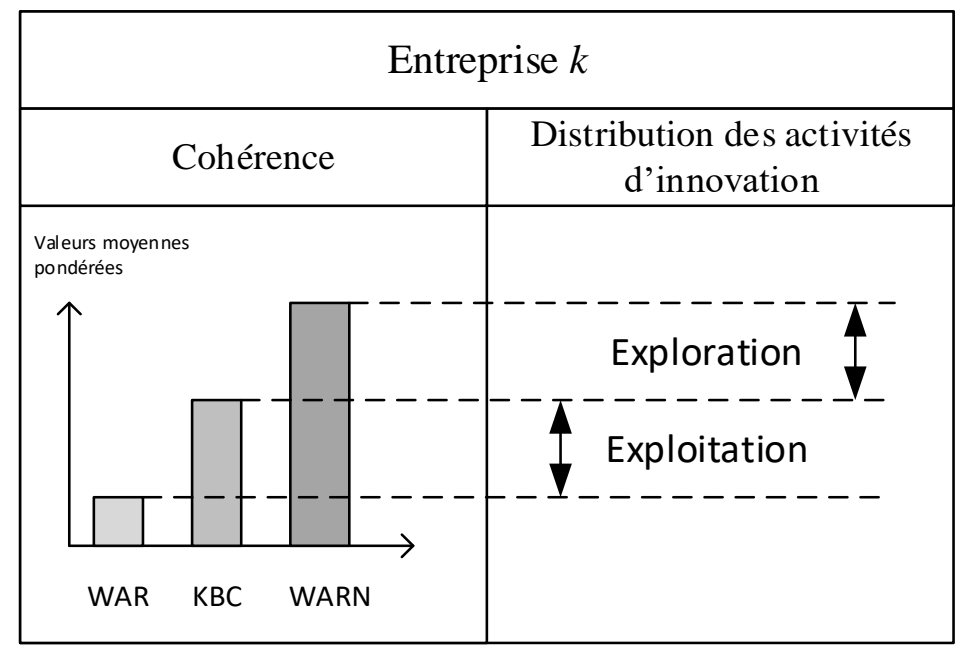

Figure 2. Exploration et exploitation dans la base de connaissance d'une entreprise

[TEE 94] montrent que WAR est nécessairement inférieur ou égal à $W A R N$. Le cas général montre que $K B C$ vient s'insérer dans cet intervalle (figure 2). La séquence $W A R \leq K B C \leq W A R N$ n'est cependant pas toujours vérifiée, notamment pour les entreprises qui manipulent des briques de connaissance déconnectées (sans relation directe et indirecte entre elles).

Nous reprenons l'idée de [ELY 15] selon qui la décision stratégique de l'entreprise en matière d'organisation de sa base de connaissance consiste en un positionnement par rapport au « cœur » des technologies qu'elle combine, i.e. par rapport à $W A R N$. L'entreprise peut en effet faire deux choix par rapport à ce cœur :

- « explorer d'étranges associations technologiques ». Ce ne sont pas celles que l'on retrouve le plus fréquemment dans l'ensemble de l'échantillon des brevets. Ces liaisons constituent en ce sens un facteur de « différenciation » pour l'entreprise ;

- «ne pas exploiter des associations technologiques évidentes ». Elles se retrouvent souvent dans l'échantillon de brevets et l'on peut envisager qu'elles contribueraient assez facilement à créer de l'innovation localement. Ne pas les exploiter est en ce sens un facteur de «distinction» pour l'entreprise.

Distinction et différenciation constituent l'activité d'exploration de l'entreprise. L'activité d'exploitation - mesurée par la différence entre $K B C$ et $W A R$ - révèle le poids du « cœur» dans l'organisation concrète de sa base de connaissance.

Les deux indicateurs relatifs sont donc :

- le taux d'exploration : EXPLOR $=(W A R N-K B C) /(W A R N-W A R)$;

- le taux d'exploitation : EXPLOIT $=1-E X P L O R=(K B C-W A R) /(W A R N-W A R)$.

La somme de leur valeur est égale à 1 .

\section{Les données : périmètre et contenu}

La population de départ est la base industrielle et technologique de défense [MIN 17]. Elle est cylindrée sur la période 2012-2014. L'analyse porte sur une sous-population de cette BITD, soit 81 
unités légales (identifiées par leur numéro SIREN). Elles ont été sélectionnées en deux temps. Le but de cette sélection est de bien centrer l'analyse sur les unités qui interviennent dans l'aéronautique, entendue dans une définition stricte comme la construction des aéronefs et leur locomotion dans l'atmosphère terrestre.

En premier lieu, ont été isolées les unités légales qui appartiennent à l'industrie manufacturière et qui fournissent le Ministère de la Défense français en 2016 pour des programmes aéronautiques militaires. L'aéronautique militaire regroupe les programmes en hélicoptères, drones, avions de combat et de transport, ainsi que l'électronique associée. Elle comprend la conception (dont la R\&D), la production et l'entretien (sans le démantèlement des équipements). Les missiles (conventionnels et balistiques) et l'espace sont exclus du champ.

Dans le second temps, un nouveau filtrage a été opéré pour conserver les fournisseurs qui appartenaient aux secteurs d'activités (APE) 2630Z (fabrication d'équipements de communication), 2651A (fabrication d'équipements d'aide à la navigation) et $3030 \mathrm{Z}$ (construction aéronautique et spatiale). Il s'agit des secteurs classiques de l'aéronautique, ce qui est confirmé par leur poids : en 2016, ils représentent $91 \%$ des paiements du Ministère de la Défense aux secteurs identifiés à la première étape de sélection (via les programmes d'aéronautique militaire).

Nous retenons deux variables extraites de la BITD. Tout d'abord le chiffre d'affaires moyen des entreprises entre 2012 et 2014 (noté CA) pour apprécier leur taille, puis une variable binaire qui traduit l'indépendance commerciale de l'entreprise à son activité militaire (notée INDEP). La valeur de 0 est attribuée à INDEP si la part du chiffre d'affaires militaire dans le chiffre d'affaires total de l'entreprise est supérieur à $20 \%$, sa valeur étant fixée à 1 sinon (la variable INDEP ne peut être traitée que discrétisée en raison de la confidentialité des données).

Nous utilisons les brevets comme proxy de l'innovation technologique. Selon [GAR 79], ils en constituent une bonne approximation. Les brevets sont des droits de propriété intellectuelle assurant une protection dans un ou plusieurs pays donnés. Il est fréquent que les inventions fassent l'objet de plusieurs demandes de brevets auprès de différents offices nationaux ou régionaux ou auprès de l'Organisation Mondiale de la Propriété Intellectuelle (WIPO), instance mondiale pour les services, les politiques, l'information et la coopération en matière de propriété intellectuelle. C'est pourquoi l'analyse en termes de familles de brevets est pertinente lorsque l'on s'intéresse à l'innovation. En effet, une famille de brevets permet de capter une innovation effective et non simplement la reproduction d'une innovation dans de multiples contextes nationaux ou régionaux. Questel, l'éditeur de la base de données ORBIT que nous utilisons, a développé un périmètre de famille qui combine la règle de famille stricte de $1^{\prime} \mathrm{OEB}^{2}$ avec des règles complémentaires qui permettent de prendre en compte les liens avec la demande parente Europe et / ou $\mathrm{PCT}^{3}$, ainsi que les liens entre les demandes américaines provisoires et les demandes américaines publiées. Ce périmètre prend également en considération les différentes définitions de la notion d'invention selon les offices.

Pour identifier les technologies contenues dans les brevets, nous utilisons la classification internationale des brevets éditée par le WIPO. Le WIPO divise la technologie en huit grandes sections ( $c f$. infra) comptants environs 70000 subdivisions au niveau le plus désagrégé.

Sur la période 2012-2014, nous avons identifié via ORBIT-Questel 3558 familles de brevets (premières dates de dépôt), composées de 10913 brevets concernant 35 des 81 entreprises de notre analyse. Ces entreprises produisent des (familles de) brevets sur l'ensemble des domaines technologiques agrégés définis par le WIPO (grandes sections ; voir figure 3), avec une dominance en technologie principale des techniques industrielles (35\%), suivies de près par les technologies relatives à la mécanique, l'éclairage, le chauffage et l'armement (31\%). Nous constatons également une forte présence des familles associées à la physique (19\%), ainsi qu'à l'électricité (11\%). 

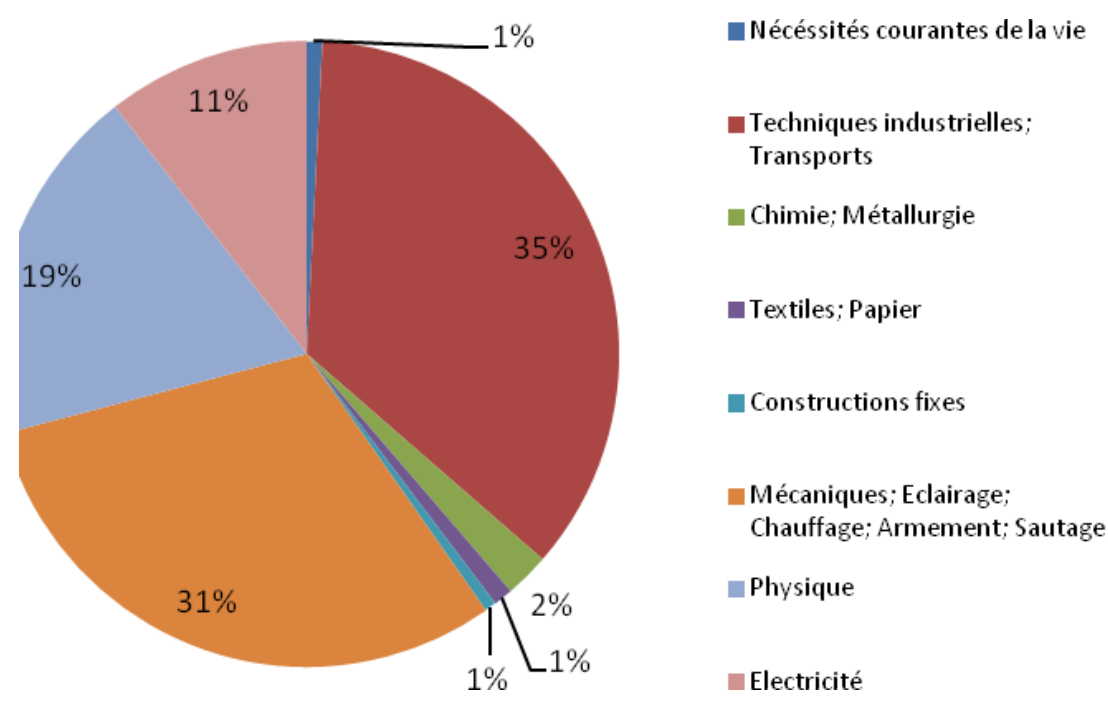

Figure 3. Répartition des familles de brevets de l'échantillon par grandes sections technologiques (domaine technologique principal)

La mesure de la cohérence technologique des entreprises nécessite de construire un paysage technologique global à l'aide d'un autre ensemble de données. Les valeurs des indices $\tau_{i j}$ sont calculées en utilisant la méthode de [NES 05] à partir de données issues de la base PATSTAT (version automne 2014) éditée par l'Office Européen des Brevets (OEB). PATSTAT est une base de données mondiale qui contient les demandes de brevets provenant de plus de 80 pays. Elle contient plus de 80 millions d'enregistrements et fournit des informations telles que les inventeurs et les propriétaires, les classes technologiques, les titres et les résumés, les offices de publication et les citations.

Nous extrayons l'ensemble des brevets demandés entre 2010 et 2012 dans les offices des brevets européen (OEB), américain (USPTO) et des pays membres de l'UE15. Nous regroupons ces brevets en familles élargies INPADOC (regroupant les brevets qui ont au moins une priorité en commun, directement ou indirectement). Le choix de la période 2010-2012 résulte de la densité des données obtenues afin de produire des paysages technologiques suffisamment riches pour avoir les indicateurs de cohérence les plus précis possibles. L'année 2010 recense ainsi plus de 327000 familles, 336000 pour 2011, et 243000 pour 2012, soit un total d'environ 907000 familles différentes.

L'indice ${ }^{\tau_{i j}}$ informe sur l'intensité de la relatedness existant entre les technologies $i$ et $i$. Plus sa valeur est élevée, plus il est commun de trouver au sein d'une même famille ces deux technologies. Chaque famille INPADOC contient l'ensemble des technologies qu'elle demande informées selon la nomenclature WIPO. Nous adoptons le découpage à 4 digits de cette nomenclature, niveau le plus communément adopté dans la littérature d'économie de l'innovation p.ex. [KRA 11]. Ce découpage permet d'identifier 643 technologies différentes, et chaque famille est une combinaison de ces technologies selon le format de la figure 1 .

Parmi ces 643 entrées technologiques, 16 font partie du sous-domaine technologique « Spatial et armement » selon [OST 10]. Ce sont ces 16 entrées que nous retenons pour lister les technologies « défense » au sein des familles extraites d'ORBIT pour notre échantillon d'entreprises (tableau 1). La part des technologies «défense » sur l'ensemble des technologies produites par les entreprises AD permettra de calculer un indicateur de dualité technologique. 
B63G Offensive or defensive arrangements on vessels, mine-laying, ...

B64G Cosmonautics, vehicles or equipment therefor (apparatus for, or methods ...

C06B Explosive or thermic compositions (blasting f42d), manufacture thereof, ...

C06C Detonating or priming devices, fuses, chemical lighters, pyrophoric ...

C06D Means for generating smoke or mist, gas-attack compositions, generation ...

C06F Matches, manufacture of matches

F41A Functional features or details common to both small arms and ordnance, ...

F41B Weapons for projecting missiles without use of explosive or combustible ...

F41C Small arms, e.g. Pistols, rifles (functional features or details common ...

F41F Apparatus for launching projectiles or missiles from barrels, e.g ....

F41G Weapon sights, aiming (optical aspects thereof g02b)

F41H Armour, armoured turrets, armoured or armed vehicles, means of attack or ...

F41J Targets, target ranges, bullet catchers

F42B

F42C

F42D

Explosive charges, e.g. For blasting, fireworks, ammunition (explosive ...

Ammunition fuses (blasting cartridge initiators f42b0003100000, chemical ...

Blasting (fuses, e.g. Fuse cords, c06c0005000000, blasting cartridges ...

Tableau 1. Les sous-classes technologiques Spatial et armement [OST 10]

Le tableau 2 présente les statistiques descriptives sur le chiffre d'affaires et le nombre de familles de brevets déposés pour l'ensemble des firmes étudiées, ainsi qu'une décomposition de celle-ci en fonction de leur niveau d'indépendance à la défense (fort ou faible). Ce tableau montre une disparité des firmes en termes de chiffre d'affaires et une relative homogénéité en termes de dépôt de familles de brevets. Si l'on compare les firmes fortement indépendantes à la défense aux firmes qui le sont faiblement, nous constatons que les premières ont un chiffre d'affaires moyen plus important que les secondes. A l'inverse, les firmes faiblement indépendantes à la défense sont moins dispersées en termes de quantité de familles de brevets déposées que les autres firmes étudiées.

\begin{tabular}{|c|c|c|c|c|c|c|c|c|c|c|c|}
\hline \multirow[b]{3}{*}{ Ensemble des firmes } & \multicolumn{6}{|c|}{ Chiffre d'affaires (en millions) } & \multicolumn{5}{|c|}{ Familles de brevets } \\
\hline & $\mathrm{N}$ & Moyenne & Ecart-type & Médiane & Min & Max & Moyenne & Ecart-type & Médiane & Min & Max \\
\hline & 81 & 525.3 & 1326.0 & 34.0 & 0.4 & 8703.8 & 52.4 & 184.3 & 0 & 0 & 1221 \\
\hline Firmes forte indépendance & 42 & 564.3 & 1615.6 & 29.2 & 0.4 & 8703.8 & 53.5 & 204.2 & 0 & 0 & 1221 \\
\hline
\end{tabular}

Tableau 2. Statistiques descriptives des firmes $A D$

Le tableau 3 présente, pour les deux types de firmes, les tests d'égalités des moyennes sur la capacité à innover, i.e. le nombre de familles déposées, et sur la taille de l'entreprise, i.e. le chiffre d'affaires. Ce tableau ne confirme qu'en partie les constations faites précédemment à partir des statistiques descriptives. En effet, ces tests montrent que les entreprises déposent en moyenne le même nombre de familles de brevets mais que la disparité des dépôts est beaucoup plus importante pour les firmes fortement indépendantes à la défense. Concernant le chiffre d'affaires, le test met en avant une homogénéité entre les deux types d'entreprises considérées.

Le tableau 4 présente les tests d'égalités des moyennes sur la taille de l'entreprise, i.e. le chiffre d'affaires, et la nature duale des activités des firmes, i.e. l'indépendance à la défense, entre les firmes déposants des brevets et les autres. Ces tests ne montrent pas de différence de moyenne entre termes d'indépendance à la défense entre les firmes innovantes et les autres. A l'inverse, nous constatons que les firmes innovantes ont un chiffre d'affaires moyen plus grand que les entreprises qui ne déposent pas de brevets. 


\begin{tabular}{|c|c|c|c|c|c|c|}
\hline Taux d'indépendance & $N$ & Moyenne & Ecart-type & Erreur Sdt & Min & $\operatorname{Max}$ \\
\hline $\mathbf{0}$ & 39 & 51.33 & 163 & 26.1 & 0 & 882 \\
\hline 1 & 42 & 53.54 & 204 & 31.51 & 0 & 1221 \\
\hline & & Egalité moyennes & P-value & $\begin{array}{c}\text { Egalité } \\
\text { variances }\end{array}$ & & \\
\hline & & oui & 0.96 & non & & \\
\hline \multicolumn{7}{|c|}{ Variable testée : Chiffre d'affaires (en millions) } \\
\hline Taux d'indépendance & $N$ & Moyenne & Ecart-type & Erreur $S d t$ & Min & $\operatorname{Max}$ \\
\hline $\mathbf{0}$ & 39 & 483.3 & 939.2 & 150.4 & 1.6 & 3500.8 \\
\hline \multirow[t]{3}{*}{1} & 42 & 564.4 & 1615.7 & 249.3 & 0.4 & 8703.9 \\
\hline & & Egalité moyennes & P-value & $\begin{array}{c}\text { Egalité } \\
\text { variances }\end{array}$ & & \\
\hline & & oui & 0.78 & oui & & \\
\hline
\end{tabular}

Tableau 3. Tests d'égalité sur brevets et chiffres d'affaires des firmes $A D$ selon la dépendance à la défense

Plus d'une entreprise sur deux (46 sur 81) ne déposent pas de brevet dans ce secteur hautement technologique. Trois raisons au moins peuvent expliquer ce phénomène :

- légalement, les entreprises fabriquant des matériels militaires ont l'obligation de déclarer à la direction générale de l'armement les demandes de brevets qu'elles déposent (article L612-8 du code de la propriété intellectuelle). La direction se prononce sur le caractère sensible de l'invention et peut exiger la non divulgation du brevet. Dans ce cas, les brevets sont mis au secret pour des raisons de souveraineté par les autorités de défense ;

- certains industriels peuvent privilégier le secret comme mode de protection de leurs inventions ;

- certaines entreprises du secteur, sous-traitants ou de maintien en condition opérationnelle, ne produisent pas d'innovations technologiques brevetables.

\begin{tabular}{|c|c|c|c|c|c|c|}
\hline \multicolumn{7}{|c|}{ Variable testée : Indépendance à la défense } \\
\hline Dépôt de famille de brevets & $N$ & Moyenne & Ecart-type & Erreur $S d t$ & Min & $\operatorname{Max}$ \\
\hline $\mathbf{0}$ & 46 & 0.57 & 0.50 & 0.07 & 0 & 1 \\
\hline 1 & 35 & 0.46 & 0.50 & 0.08 & 0 & 1 \\
\hline & & Egalité moyennes & $p$-value & Egalité variances & & \\
\hline & & oui & 0.34 & non & & \\
\hline \multicolumn{7}{|c|}{ Variable testée : Chiffre d'affaires (en millions) } \\
\hline Dépôt de famille de brevets & $N$ & Moyenne & Ecart-type & Erreur Sdt & Min & $\operatorname{Max}$ \\
\hline $\mathbf{0}$ & 46 & 123.7 & 2690.4 & 39.6 & 0.4 & 1375.9 \\
\hline \multirow[t]{3}{*}{1} & 35 & 1053.1 & 1880.7 & 317.8 & 1.8 & 8703.9 \\
\hline & & Egalité moyennes & $p$-value & Egalité variances & & \\
\hline & & non & 0.0014 & oui & & \\
\hline
\end{tabular}

\section{Principaux résultats}

En synthèse, les relations et variables qui définissent les profils des entreprises AD dans notre étude sont :

- relation 1 (R1): la dualité technologique (IPCNONDEF) et la dualité productive (INDEP). IPCNONDEF prend la forme d'un ratio rapportant le nombre de technologies non défense développées par l'entreprise selon la nomenclature de [OST 10] sur son nombre total de technologies développées. INDEP est une variable booléenne indiquant le poids du chiffre d'affaires non défense de l'entreprise. Si au plus $20 \%$ de son chiffre d'affaires (en moyenne sur 2012-2014) est issu de la sphère militaire, la variable INDEP prend la valeur 1. Elle prend la valeur 0 sinon. Par hypothèse, ces deux variables sont censées agir dans le même sens pour définir le profil des entreprises AD (cf. section 1); 
- relation 2 : la diversité technologique (DIV) et le ratio d'exploration (EXPLOR). DIV indique l'étendue de la maîtrise technologique de l'entreprise - le nombre de postes différents de la nomenclature WIPO à 4 chiffres des brevets qu'elle produit sur la période 2012-2014. EXPLOR est une variable de cohérence qui reflète l'importance des comportements stratégiques de différenciation et de distinction technologiques de l'entreprise. Par hypothèse, DIV et EXPLOR sont censées agir dans le même sens pour définir le profil des entreprises AD [QUI 08] si l'on postule que ces entreprises se comportent comme les autres ;

- les variables PATENT (nombre de brevets déposés sur 2012-2014), IPC (nombre de technologies développées durant cette même période) et CA (chiffre d'affaires moyen sur 2012-2014) sont prises en compte pour contrôler ces relations. PATENT et IPC sont retenues car, ensemble, elles informent sur l'étendue technologique des brevets déposés par l'entreprise. CA est l'une des caractéristiques non technologiques essentielle de l'entreprise : sa taille.

La figure 4 reproduit les résultats d'une double analyse de données: analyse factorielle et classification ascendante hiérarchique. Chaque entreprise est représentée par un vecteur comprenant sept valeurs, pour chacune des variables décrites précédemment. Tout d'abord, une analyse factorielle projette ces vecteurs dans un plan, et la contribution de chaque variable aux deux principaux facteurs est indiquée. Ensuite, une classification ascendante hiérarchique utilisant des distances pondérées moyennes permet d'identifier cinq classes d'entreprises (en couleur dans la figure 4).

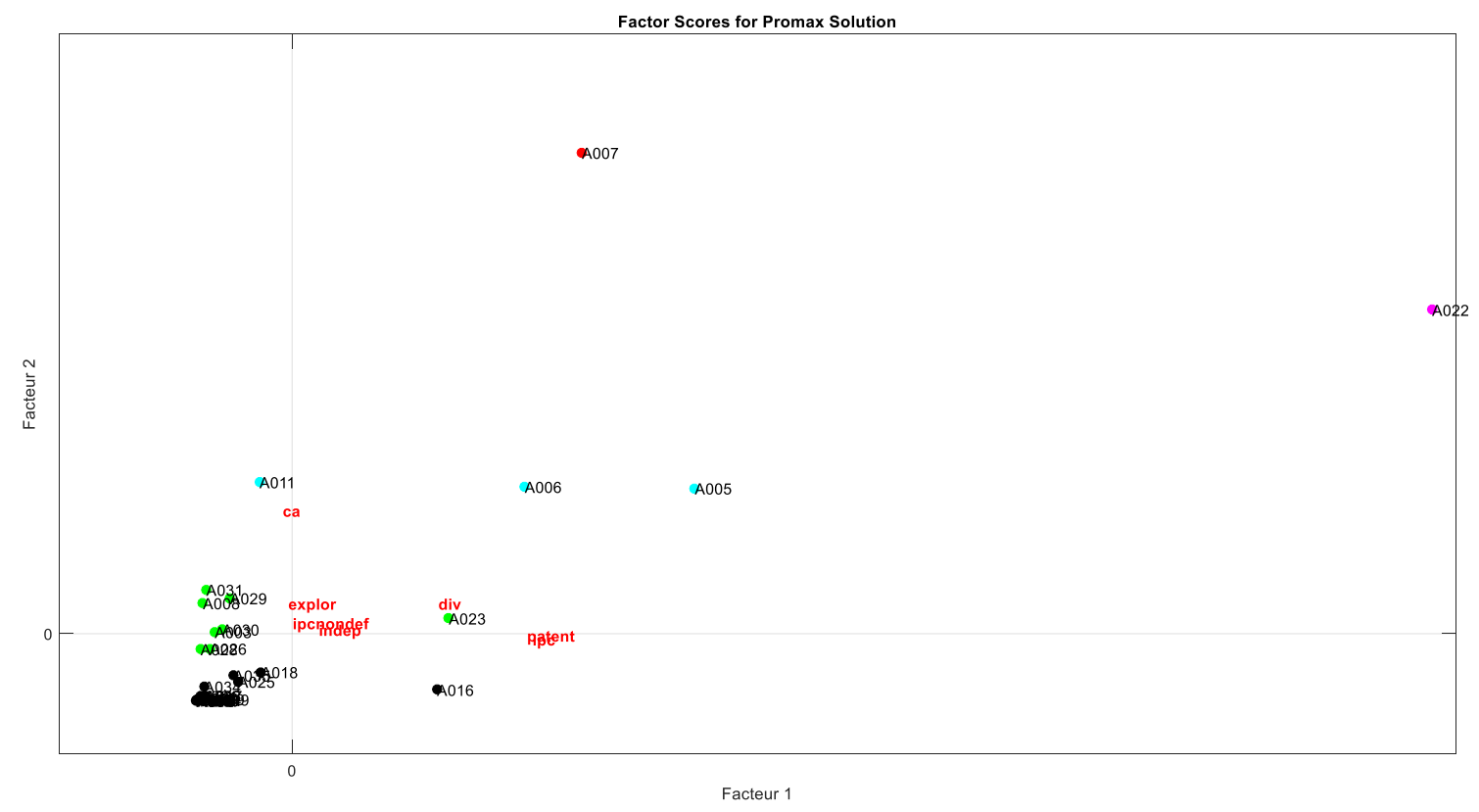

Figure 4. Analyse factorielle sur la cohérence technologique des entreprises aéronautiques de défense

Les variables technologiques de contrôle (PATENT, IPC) contribuent fortement à définir le premier facteur, alors que la variable de contrôle non technologique (CA) participe activement au second. En ce qui concerne la relation R2, la variable DIV s'associe positivement aux deux facteurs, la variable EXPLOR positivement au deuxième uniquement. Diversité technologique et ratio d'exploration (la figure 5 fait apparaître la forme prise par cette relation sur notre échantillon) vont donc en partie dans le sens de l'étendue technologique et de la taille de l'entreprise. Par contre, la relation R1 ne se vérifie pas : IPCNONDEF contribue au deuxième facteur (et s'associe donc à la taille) alors que INDEP ne contribue à aucun facteur. 


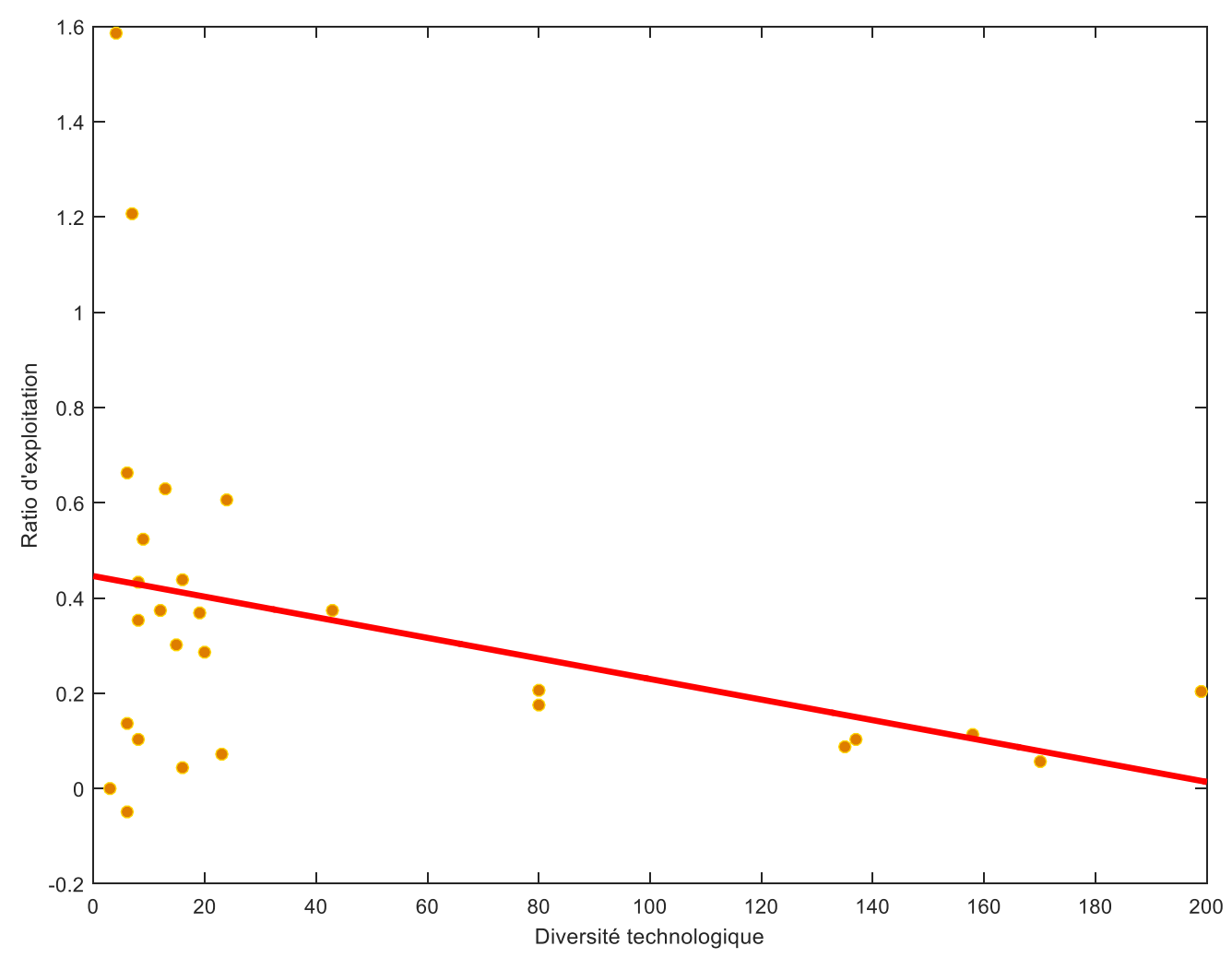

Figure 5. Diversité technologique et ratio d'exploitation

La relation R1 entre les deux dualités (technologique et productive) n'est pas établie pour les entreprises AD. Cela peut être dû en partie au fait que nos indicateurs de dualité peuvent être discutés, à la fois dans leurs formes et dans les seuils que nous avons retenus. Concernant leurs formes, nous avons justifié dans la section 2 un indicateur prenant pour base les technologies strictement « défense » de la nomenclature maintenue par [OST 10]. Sans doute cette base technologique (tableau 1) est-elle trop étroite, faisant que le ratio IPCNONDEF prend une valeur trop élevée en moyenne. Pour l'indicateur INDEP, le choix de passer par une variable booléenne est discutable : si le seuil de $20 \%$ maximum de chiffre d'affaires « militaire » pour affecter une valeur de 1 à cette variable est sans doute raisonnable, passer par une variable continue plutôt que binaire aurait peut-être permis d'obtenir des résultats plus riches.

Dans le domaine de l'aéronautique de défense, des entreprises à très forte diversité cohabitent avec des pure players aux marchés plus étroits et aux productions plus spécifiques. Pour la relation R2, la croissance du taux d'exploration avec la diversité technologique fait écho à l'approche de [QUI 08] sur plusieurs plans. Ils posent en effet que si la diversité technologique a un effet plus important sur la capacité exploratoire que sur la capacité d'exploitation de l'entreprise, une entreprise possédant une base technologique diversifiée dispose également d'un potentiel innovant supérieur. De plus, cette diversité peut atténuer les rigidités et les dépendances de sentier en accélérant le «taux d'invention » de l'entreprise, en particulier lorsque l'on s'écarte des activités routinières de celle-ci. Cela ouvre des perspectives nouvelles pour l'étude spécifique des profils d'innovation des entreprises, notamment dans le secteur AD.

La plus directe de ces perspectives est, selon nous, issue des travaux de [ZYL 17]. Elle part de l'approche de Quintana-Garcia et Benavides-Velasco en développant une représentation dans laquelle la capacité d'une entreprise à s'écarter de ses routines se traduit en un «potentiel de distorsion » révélé par l'écart entre « les » cœurs technologiques de l'entreprise, d'une part lorsque ce cœur est défini sur sa seule activité principale et d'autre part lorsqu'il est défini sur le paysage technologique global. En 
retenant l'approche empirique de la cohérence technologique de l'entreprise décrite en section 3, cela revient à produire des indicateurs $C_{k}$ différenciés lorsque la base des brevets pour mesurer les indices $\tau_{i j}$ se centre dans un premier temps sur les seules entreprises du secteur principal de l'entreprise (AD, par exemple) et dans un deuxième temps sur toutes les entreprises indépendamment de leur secteur principal. Lorsqu'une entreprise développe une liaison technologique appartenant au cœur global sans qu'elle soit du cœur sectoriel, elle innove au-delà de sa compétence technologique principale, et elle acquière la capacité à combiner les cœurs de façon à produire des innovations de rupture pour son secteur de base. Zyla montre empiriquement - notamment pour les entreprises du secteur aéronautique - que le potentiel de distorsion est positivement lié à la diversité technologique de l'entreprise. Ce résultat donne corps à la proposition selon laquelle il existe une relation positive entre diversité technologique et taux d'invention en lui attribuant un contenu empirique précis.

\section{Conclusion}

Cette étude de la $R \& D$ des entreprises AD de la BITD française a permis de mettre en évidence deux éléments majeurs. D'une part, l'étude ne valide pas l'hypothèse d'une relation positive entre la dualité technologique et la dualité productive dans ce secteur. Cela met en lumière la faiblesse de la classification trop restrictive des technologies Spatial et armement qui ne prend pas en considération, par exemple, l'ensemble des technologies de radar (codes WIPO à trois digits G01 et G09) qui sont essentielles pour l'activité $\mathrm{AD}$. D'autre part, notre deuxième axe de recherche concernant les stratégies de $R \& D$ approchées par la relation entre la diversité technologique et le ratio d'exploration est plus prometteur. En effet, notre analyse en termes de relatedness technologique montre une relation positive entre le nombre de technologies maitrisées par les entreprises et la cohérence reflétée par les comportements d'« exploration d'étranges associations technologiques» (différenciation) et d'«absence d'exploitation d'associations technologiques évidentes» (distinction). Dans l'avenir, il serait intéressant d'analyser les profils des entreprises $\mathrm{AD}$ dans une perspective comparative à l'international dans un contexte marqué par de récents rebondissements sur ce marché : émergence de nouveaux concurrents, notamment chinois (Commercial Aircraft Corporation of China), repositionnement commercial d'Airbus autour des aéronefs civils, etc.

\section{Bibliographie}

[ANS 65] ANSOFF H.I., Stratégie du développement de l'entreprise, Edition Hommes et Techniques (traduction française, 1981), Paris, 1965.

[AYE 08] Ayerbe C., LAZARic N., CAllois M., Mitkova L., « Nouveaux enjeux d'organisation de la propriété intellectuelle dans les industries complexes : une discussion à partir du cas de Thales », Revue d'Economie Industrielle, $\mathrm{n}^{\circ} 137$, p. 9-42, 2008.

[BEL 08] BELIN J., GUILLE M., «R\&D et innovation: quel financement pour les entreprises défense ? », Innovations, $\mathrm{n}^{\circ} 28$, p. 33-59, 2008.

[BOT 10] BotTAZZI G., PIRINO D., « Measuring industry relatedness corporate coherence », LEM Working Paper Series, 2010.

[CHR 81] CHRISTENSEN H.K., MONTGOMERY C.A., « Corporate economic performance: diversification strategy versus market structure », Strategic Management Journal, vol. 2, p. 327-343, 1981.

[ELY 13] EL Younsi H., Les stratégies de recentrage des entreprises : Europe, États-Unis, 1990-2008, Presses Académiques Francophones, Paris, 2013.

[ELY 14] EL YOUNSI H., LEBERT D., «Un retour au ratio de spécialisation pour interpréter les stratégies de développement des entreprises non financières : Europe et États-Unis, 1992-2007 », Economie Appliquée, vol. 67, $\mathrm{n}^{\circ} 1$, p. 5-36, 2014.

[ELY 15] El YOUNSI H., LEBERT D., MEUNIER F.-X., ZYLA C., « Exploration, exploitation et cohérence technologique », Economie Appliquée, vol. 68, n 3, p. 187-204, 2015. 
[FAN 00] FAN J., LANG L., « The measurement of relatedness: an application to corporate diversification », The Journal of Business, vol. 73, p. 629-660, 2000.

[FON 14] Fonfría A., DUCh-BRown N., «Explaining export performance in the Spanish defense industry », Defence and Peace Economics, vol. 25, p. 51-67, 2014.

[GAR 79] GARFIELD E., «Is citation analysis a legitimate evaluation tool? », Scientometrics, vol. 1, p. 359-375, 1979.

[KLE 09] KleIN P.G., KLEIN S.K., LIEN L.B., «Are divestitures predictable? A duration analysis », Working paper, Contracting and Organizations Research Institute, University of Missouri, 2009.

[KRA 11] KRAFFT J., QUATRARo F., SAVIOTTI P.P., « The knowledge base evolution in biotechnology: a social network analysis », Economics of Innovation and New Technology, vol. 20, p. 445-475, 2011.

[MAR 91] MARCH J.G., «Exploration and exploitation in organizational learning », Organization Science, vol. 2, Special Issue: Organizational Learning: Papers in. Honor of (and by) James G. March, p. 71-87, 1991.

[MIN 13] MinisTERE DE LA DEFENSE, Livre blanc sur la défense nationale et la sécurité, Edition numérique, 2013.

[MIN 17] MinisTERE DE LA DEFENSE, Annuaire statistique de la défense, 2017.

[MON 79] MONTGOMERY C., Diversification, market structure, and firm performance: an extension of Rumelt's model, Unpublished doctoral dissertation, Purdue University, 1979.

[MON 94] MONTGOMERY C.A., «Corporate diversification », The Journal of Economic Perspectives, vol. 8, p. 163-178, 1994.

[NAS 13] NASIRIYAR M., NeSTA L., DiBIAGgIO L., « The moderating role of the complementary nature of technological resources in the diversification-performance relationship », Industrial and Corporate Change, doi:10.1093/icc/dtt058, 2013.

[NES 05] NeSTA L., SAVIOTTI P.P., « Coherence of the knowledge base and the firm's innovative performance: evidence from the U.S. pharmaceutical industry », Journal of Industrial Economics, vol. 53, p. 123-142, 2005.

[OST 10] OST, Indicateurs de sciences et de technologies, Rapport de l'Observatoire des Sciences et des Techniques, Paris, 2010.

[OUD 15] OUdot J.-M., QUEMENER J., «Les dépenses de R\&D de la BITD : une évaluation par le crédit impôt recherche », Ecodef, n ${ }^{\circ}$ 74, Ministère de la Défense, 2015.

[PAL 85] PALEPU K., «Diversification strategy, profit performance and the entropy measure », Strategic Management Journal, vol. 6, p. 239-255, 1985.

[PIS 00] PISCITELLO L., « Relatedness and coherence in technological and product diversification of the world's largest firms », Structural Change and Economic Dynamics, vol. 11, p. 295-315, 2000.

[PIS 04] PISCITELLO L., « Corporate diversification, coherence and economic performance », Industrial and Corporate Change, vol. 13, p. 757-787, 2004.

[QUI 08] QUINTANA-GARCIA C., BENAVIDES-VElasCo C.A. (2008), «Innovative competence, exploration and exploitation: the influence of technological diversification », Research Policy, vol. 37, p. 492-507, 2008.

[ROB 95] RoBINS J., WIERSEMA M.F., «A resource-based approach to the multibusiness firm: empirical analysis of portfolio interrelationships and corporate financial performance », Strategic Management Journal, vol. 16, p. 277-299, 1995.

[RON 05] RONDI L., VANNONI D., «Are EU leading firms returning to core business? Evidence on refocusing and relatedness in a period of market integration », Review of Industrial Organization, vol. 27, p. 125-145, 2005.

[RUM 74] RUMELT R.P., Strategy, structure, and economic performance, Harvard University Press, 1974.

[RUM 82] RUMELT R.P., « Diversification strategy and profitability », Strategic Management Journal, vol. 3, p. 359-369, 1982.

[SAV 09] SAVIOTTI P.P., « Knowledge networks: structure and dynamics », dans A. PYKA, A. SchaRnhORST (eds.), Innovation networks: understanding complex systems, Springer-Verlag, Berlin, 2009.

[SCH 82] SCHERER F.M., «Inter-industry technology flows and productivity growth », The Review of Economics and Statistics, vol. 64, p. 627-634, 1982.

[SER 08] SERFATI C., «Le rôle de l'innovation de Défense dans le système national d'innovation de la France », Innovations, $\mathrm{n}^{\circ}$ 28, p. 61-83, 2008. 
[SER 14] SERFATI C., L'industrie française de défense, La documentation française, Paris, 2014.

[TAN 05] TANRIVERDI H. VENKATRAMAN N., «Knowledge relatedness and the performance of multibusiness firms », Strategic Management Journal, vol. 26, p. 97-119, 2005.

[TEE 94] TEECE D.J, RUMELT R., Dosi G., WINTER S., «Understanding corporate coherence: theory and evidence », Journal of Economic Behavior and Organization, vol. 23, p. 1-30, 1994.

[ZYL 17] ZYLA C., Ruptures technologiques, thèse de doctorat, Université Paris 1 Panthéon-Sorbonne, 2017.

\footnotetext{
${ }^{1} 2630 \mathrm{Z}$ (Fabrication d'équipements de communication), 2651A (Fabrication d'équipements d'aide à la navigation) et $3030 \mathrm{Z}$ (Construction aéronautique et spatiale).
}

${ }^{2}$ L'office Européen des Brevets (OEB) définit une famille de brevets comme comprenant tous les documents ayant la même priorité ou la même combinaison de priorités.

${ }^{3}$ «Le Traité de coopération en matière de brevets (PCT) aide les déposants à obtenir une protection par brevet au niveau international, aide les offices de brevets dans leurs décisions d'octroi de brevets, et facilite l'accès du public à une mine d'informations techniques relatives à ces inventions. En déposant une seule demande internationale de brevet selon le PCT, les déposants peuvent demander la protection d'une invention simultanément dans 148 pays à travers le monde » (http://www.wipo.int/pct/fr/, consulté le 03/08/2016). 